\title{
Optical spectroscopic sensing of tumor hypoxia
}

Sina Dadgar

Joel Rodríguez Troncoso

Narasimhan Rajaram 


\title{
Optical spectroscopic sensing of tumor hypoxia
}

\author{
Sina Dadgar, Joel Rodríguez Troncoso, and Narasimhan Rajaram* \\ University of Arkansas, Department of Biomedical Engineering, Fayetteville, Arkansas, United States
}

\begin{abstract}
Tumor hypoxia is a critical indicator of poor clinical outcome in patients with cancers of the breast, cervix, and oral cavity. The ability to noninvasively and reliably monitor tumor oxygenation both prior to and during therapy can aid in identifying poor treatment response earlier than is currently possible and lead to effective changes in treatment regimen. Diffuse reflectance spectroscopy (DRS) has been used in several studies to measure tissue scattering, total hemoglobin content $(\mathrm{THb})$, and vascular oxygenation $\left(\mathrm{sO}_{2}\right)$ in tissue. In this study, we validate in vivo DRS-based measurements of vascular oxygenation using immunohistochemical staining of tumor hypoxia using pimonidazole, an established hypoxia marker. Using tumor xenografts grown from two different head and neck cell lines-UM-SCC-22B and UM-SCC-47-we demonstrate statistically significant negative correlations between tumor hypoxic fraction (HF) and $\mathrm{THb}(r=-0.45 ; p=0.04)$ and $\mathrm{sO}_{2}(r=-0.50$; $p=0.02)$. In addition, we also found a statistically significant positive correlation between $\mathrm{HF}$ and mean reduced scattering coefficient $(r=0.60 ; p=0.005)$. Our results demonstrate that DRS-based measures of $\mathrm{sO}_{2}$ can provide reliable indirect measurements of tumor hypoxia that can be of significant utility in preclinical and clinical studies. ( ) The Authors. Published by SPIE under a Creative Commons Attribution 3.0 Unported License. Distribution or reproduction of this work in whole or in part requires full attribution of the original publication, including its DOI. [DOI: 10.1117/1.JBO.23.6.067001]
\end{abstract}

Keywords: hypoxia; diffuse reflectance; vascular oxygenation.

Paper 180092R received Feb. 12, 2018; accepted for publication May 17, 2018; published online Jun. 5, 2018.

\section{Introduction}

Low oxygen tension or hypoxia is caused by the imbalance between oxygen delivery and consumption. ${ }^{1-4}$ Hypoxia is typically defined at the cellular level as oxygen tension $\left(\mathrm{pO}_{2}\right)<10 \mathrm{mmHg}$. Oxygen-sensing Clark microelectrodes have established a wealth of knowledge related to hypoxia and its role in poor disease-free survival. ${ }^{5-7}$ These electrodes provide a direct measure of $\mathrm{pO}_{2}$ and hence hypoxia in tissue. To measure oxygen tension in vivo, the electrode is inserted into tissue to a certain depth and retracted along tracks to provide $\mathrm{pO}_{2}$ measurements at several points along these tracks. Such measurements of hypoxia have been associated with tumor aggressiveness, resistance to chemotherapy and radiation therapy, ${ }^{4,8-11}$ and shown to be predictive of tumor metastases in soft tissue sarcoma. ${ }^{12}$ It has also been reported that local recurrences have a higher hypoxic fraction (HF) than primary tumors. ${ }^{13,14}$ These studies have demonstrated that measuring tumor oxygenation either prior to or during therapy could aid in the selection of appropriate treatment regimens for patients. However, microelectrode-based $\mathrm{pO}_{2}$ measurements are always invasive (requiring tumor penetration), limited to only accessible organs, can potentially modify the tumor microenvironment, and are not amenable to repeated measurements in vivo. A combination of pulsed electron paramagnetic resonance imaging and magnetic resonance imaging has been shown to provide quantitative three-dimensional maps of tumor $\mathrm{pO}_{2}$ co-registered with anatomical detail in preclinical animal models. ${ }^{15}$ Other than an intravenous injection of the triarylmethyl radical (TAM) probe used to sense oxygen, this technology would allow noninvasive and repeated imaging of $\mathrm{pO}_{2}$

*Address all correspondence to: Narasimhan Rajaram, E-mail: nrajaram@uark .edu in vivo. However, the instrumentation in its current form presents a challenge to clinical translation.

Other established methods to measure tumor hypoxia include the use of pimonidazole $e^{6,7,16,17}$ and EF5, ${ }^{18}$ two widely used markers that provide quantitative measures of tumor hypoxia. Both pimonidazole and EF5 accumulate by forming adducts with thiol groups present in proteins in hypoxic cells. Pimonidazole accumulates in cells with $\mathrm{pO}_{2}<10 \mathrm{mmHg}$ and has been used extensively to map hypoxic regions in excised tumors in animal models. EF5 has been demonstrated in both animal and human studies (using a fluorinated form of EF5) and can be calibrated to provide information about both location and level of hypoxia. ${ }^{19}$ Since quantification using these markers requires either tumor excision after administration or the use of radioisotopes for in vivo labeling, it precludes their use in longitudinal animal and human studies, where tumor oxygenation could potentially be used as a biomarker of treatment response or local recurrence.

For accessible tumors of the skin and oral cavity, optical fiber-based diffuse reflectance spectroscopy (DRS) can provide a nondestructive and noninvasive alternative to the microelectrode for quantitatively evaluating tumor oxygenation. The tissue of interest is illuminated with visible light from a broadband source (400 to $650 \mathrm{~nm}$ ) and the reflected light is used to quantify the underlying scatterers and absorbers, a combination of which is used for recognition of tissue pathology. Tumor vascular oxygenation $\left(\mathrm{sO}_{2}\right)$ can be quantified by measuring the individual contributions of oxygenated $\left(\mathrm{HbO}_{2}\right)$ and deoxygenated $(\mathrm{dHb})$ hemoglobin within the blood vessels. Thus, DRS provides an indirect measure of tissue $\mathrm{pO}_{2}$ by quantifying the "supply"oxygenation levels in the vasculature using $\mathrm{sO}_{2}$ and perfusion levels using total hemoglobin concentration $\left(\mathrm{HbO}_{2}+\mathrm{dHb}\right)$. DRS has previously been used to measure tumor oxygenation in small animal model studies to determine treatment response. 
Vishwanath et al. ${ }^{20}$ found that longitudinal changes in $\mathrm{dHb}$, as quantified by DRS, after treatment with doxorubicin were concordant with changes in tumor HF. Palmer et al. ${ }^{21}$ demonstrated that DRS-based measurements of $\mathrm{sO}_{2}$ were more stable compared with microelectrode-based measurements of $\mathrm{pO}_{2}$; furthermore, the direction of change of $\mathrm{sO}_{2}$ in response to perturbations in the composition of inhaled air in mice was concordant with $\mathrm{pO}_{2}$ measurements. This study builds upon these previous reports to validate DRS measures of $\mathrm{sO}_{2}$ using pimonidazolebased quantification of tumor HF in two human head and neck cancer cell-based xenografts. In addition to evaluating $\mathrm{HF}$ at a depth corresponding to the sampling depth of our probe, we also determined the HF at two other depths within excised tissue and compared these measurements to $\mathrm{sO}_{2}$. Our results indicate that DRS-based measurements of $\mathrm{sO}_{2}$ can provide reliable estimates and thus enable longitudinal tracking of tumor HF in studies, where tumor hypoxia can be a significant biomarker of treatment response.

\section{Materials and Methods}

\subsection{Cell Culture and Tumor Xenografts}

All studies and protocols were approved by the Institutional Animal Care \& Use Committee (IACUC) of University of Arkansas. Twenty nude mice were housed at the Central Laboratory Animal Facility (CLAF). Mice were maintained under standard 12-h light/dark cycles with ad libitum access to food and water. Mice were subcutaneously injected with either UM-SCC-47 $(n=10)$ or UM-SCC-22B $\quad(n=10)$ human head and neck cancer cells. Cells were cultured in a mixture of Dulbecco's modified Eagle medium, 10\% fetal bovine serum, $1 \%$ Penicillin-Streptomycin, $1 \%$ nonessential amino acids, and $1 \%$ L-glutamine. Head and neck tumor xenografts were formed by injecting 1.5 million cells suspended in $1: 1$ mixture of Matrigel (Corning, New York) and saline into the right flank of these animals. When tumor volumes reached $1500 \mathrm{~mm}^{3}$, mice received an intraperitoneal injection of pimonidazole (at a dose of $60 \mathrm{mg} / \mathrm{kg}$ ). An hour postinjection, the animals were anesthetized by breathing $1.5 \%$ isoflurane (mixed with oxygen) and diffuse reflectance spectra were acquired from each tumor. Immediately after DRS measurements, the tumors were resected (with skin) and snap-frozen for histology.

\subsection{Diffuse Reflectance Spectroscopy}

Figure 1(a) presents our DRS setup. This simple and portable system consists of a halogen lamp (HL-2000, Ocean Optics, Dunedin, Florida) for illumination, a USB spectrometer (Flame, Ocean Optics) for spectral acquisition, and a bifurcated optical probe for light delivery and collection. DRS spectra were acquired in the wavelength range of 475 to $600 \mathrm{~nm}$. The common end of the probe is illustrated in Fig. 1(b) and consists of four illumination fibers and five detector fibers located at a source-detector separation distance (SDSD) of $2.25 \mathrm{~mm}$. Based on previously described methods, we determined the sampling depth of this SDSD to be $\sim 1.8 \mathrm{~mm} .^{22}$ Reflected light spectra were collected using this system with an integration time of $100 \mathrm{~ms}$. Spectral acquisition was simplified with the use of a foot pedal controlled by custom LabVIEW (National Instruments, Austin, Texas) software. With the exception of three tumors on which multiple spectra were recorded, only

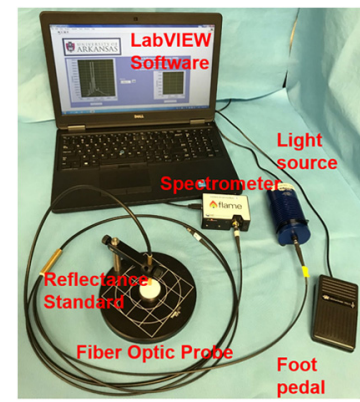

(a)

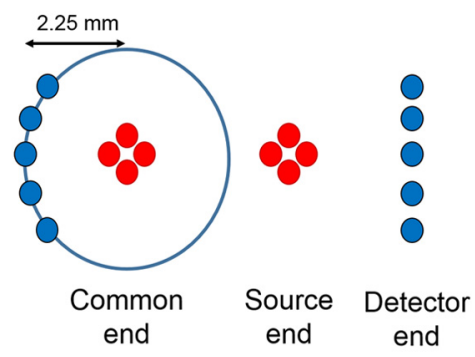

(b)
Fig. 1 (a) Experimental DRS setup and (b) bifurcated fiber optic probe illustrating the common tissue end, the source end that is connected to the light source, and the detector end that is connected to the USB spectrometer.

one spectrum was measured from each tumor. For tumors with multiple spectra, averaged optical properties were used to represent that tumor. Spectra acquired from tissues were background-subtracted to correct for dark current and ambient light. To calibrate for light throughput, the background-subtracted reflected light intensity from tissue was divided by background-subtracted reflected light intensity from an $80 \%$ reflectance standard (SRS-80-010; Labsphere, North Sutton, New Hampshire).

\subsection{Quantification of Tissue Optical Properties}

We used a lookup table (LUT)-based model to fit the acquired DRS spectra and extract wavelength-dependent absorption and scattering properties from tissue. The LUT-based model has been described in detail previously ${ }^{23}$ and validated for a range of SDSD. ${ }^{22}$ Briefly, the LUT is generated from reflectance spectra acquired from a matrix of tissue-simulating phantoms of known absorption $\left(\mu_{\mathrm{a}}\right)$ and scattering $\left(\mu_{\mathrm{s}}^{\prime}\right)$ coefficients. These phantoms contained India ink (Salis International Inc., Golden, Colorado) and polystyrene microspheres beads (diameter $=1 \mu \mathrm{m}$; Polysciences, Warrington, Pennsylvania) of known concentrations dissolved in water to create a range of absorption and scattering coefficients, respectively. The LUT corresponding to the SDSD used in this study was validated using tissue-simulating phantoms and found to have errors of $9.5 \%$ and $1.5 \%$, respectively, for quantifying $\mu_{\mathrm{a}}$ and $\mu_{\mathrm{s}}^{\prime}$, respectively. To fit the model to the data, we constrained scattering to a power law dependence on wavelength and represented it by the equation: $\mu_{\mathrm{s}}^{\prime}(\lambda)=\mu_{\mathrm{s}}^{\prime}\left(\lambda_{0}\right) \cdot\left(\frac{\lambda}{\lambda_{0}}\right)^{-B}$, where $\lambda_{0}=630 \mathrm{~nm}$. The absorption coefficient is computed as a linear sum of absorbers based on Beer-Lambert's law. Assuming the principal absorbers to be $\mathrm{HbO}_{2}, \mathrm{dHb}$, and mouse skin, the absorption coefficient is given by $\mu_{\mathrm{a}}(\lambda)=[\mathrm{Hb}]\left[\alpha \sigma_{\mathrm{HbO}_{2}}(\lambda)+(1-\alpha) \sigma_{\mathrm{Hb}}(\lambda)\right]+$ $[C] \operatorname{skin}(\lambda)$, where $\alpha$ is the vascular oxygenation and $\sigma$ is the extinction coefficient of hemoglobin (oxygenated or deoxygenated). We have previously shown that the effects of pigment packaging, which describes the inhomogeneous distribution of hemoglobin within tissue, are minimal at wavelengths beyond $500 \mathrm{~nm} .{ }^{24}$ Therefore, we did not include a correction factor to account for pigment packaging.

\subsection{Immunohistochemistry}

All harvested tumors were sectioned using a Cryostat (CM 1860; Leica, Inc., Nussloch, Germany) to slices of $10 \mu \mathrm{m}$. 
Care was taken to slice the frozen tumors starting from the skin side, where the DRS measurements were initiated. Multiple slices were acquired from each tumor at depths corresponding to $0.8,1.8$, and $2.8 \mathrm{~mm}$, with separate, sequential slices utilized for immunofluorescence and histology. All of the collected samples were immunostained with a direct labeling protocol. First, the slides were hydrated in phosphate-buffered saline (PBS) and permeabilized with $0.1 \%$ Triton-X. Next, the slides were incubated with blocking solution (95\% PBS, $4 \%$ goat serum, and $1 \%$ sodium azide) in room temperature for an hour and stained with mouse monoclonal antibody conjugated to Dylight ${ }^{\mathrm{TM}} 549$ fluorophore (Hypoxyprobe Red549 kit; HPI, Inc., Burlington, Massachusetts) for an hour in a dark room. Finally, slides were rinsed with PBS, covered with fluoromount, and coverslipped with nail polish. Slides were dried for $24 \mathrm{~h}$ prior to imaging. All images were acquired using a $4 \times$ objective on a Nikon fluorescence microscope with same settings (binning, exposure time, and gain).

\subsection{Quantification of Hypoxic Fraction and Image Analysis Algorithm}

Pimonidazole accumulates in tissue, where the intracellular oxygen level drops below $10 \mathrm{mmHg} .{ }^{25}$ We analyzed all of the acquired immunofluorescence images using MATLAB (Mathworks; Natick, Massachusetts). At each depth within the tumor-0.8, 1.8, and $2.8 \mathrm{~mm}$ [Fig. 2(a)]—immunofluorescence and histology (H\&E-stained) images were acquired from multiple fields of view from each tumor slice. For representative purposes alone, a fully stitched image of pimonidazole immunofluorescence is presented in Fig. 2(b) along with its corresponding H\&E image from an adjacent tumor slice. Each tumor slice consisted of an average of 15 fields of view $(1.5 \mathrm{~mm} \times 1.1 \mathrm{~mm})$. We converted each individual image to its gray-scaled and normalized version and calculated a threshold using Otsu's method to segment out the pimonidazole-positive pixels. ${ }^{26}$ Next, we averaged all thresholds to compute a global threshold. This global threshold was used to segment all of the images, and $\mathrm{HF}$ of each image was quantified as the ratio of hypoxic (segmented) pixels to the total number of tissue pixels. Figure 2(c) presents a flowchart of the image processing algorithm employed for this analysis. The average HF corresponding to each depth within a tumor is reported here.

\section{Results and Discussion}

Figure 3(a) presents DRS spectra and their corresponding LUT fits (red lines) for two different tumors that were excised at similar tumor volumes. Quantification of optical properties using the LUT fits identified different values of $\mathrm{sO}_{2}$ for each tumor. This is evident in the wavelength-dependent absorption coefficients for each tumor, which demonstrate the classic double peaks of $\mathrm{HbO}_{2}(542$ and $577 \mathrm{~nm})$ for the tumor with recovered $\mathrm{sO}_{2}$ of $58 \%$. On the other hand, the $\mu_{\mathrm{a}}$ spectrum for the tumor with $\mathrm{sO}_{2}$ of $15 \%$ indicates poor oxygenation with a single peak corresponding to $\mathrm{dHb}$ [Fig. 3(b)]. IHC images of HF corresponding to both tumors illustrate that the tumor with higher $\mathrm{sO}_{2}$ has a lower percentage of hypoxic tissue compared with the low-sO $\mathrm{O}_{2}$ tumor [Fig. 3(c)].

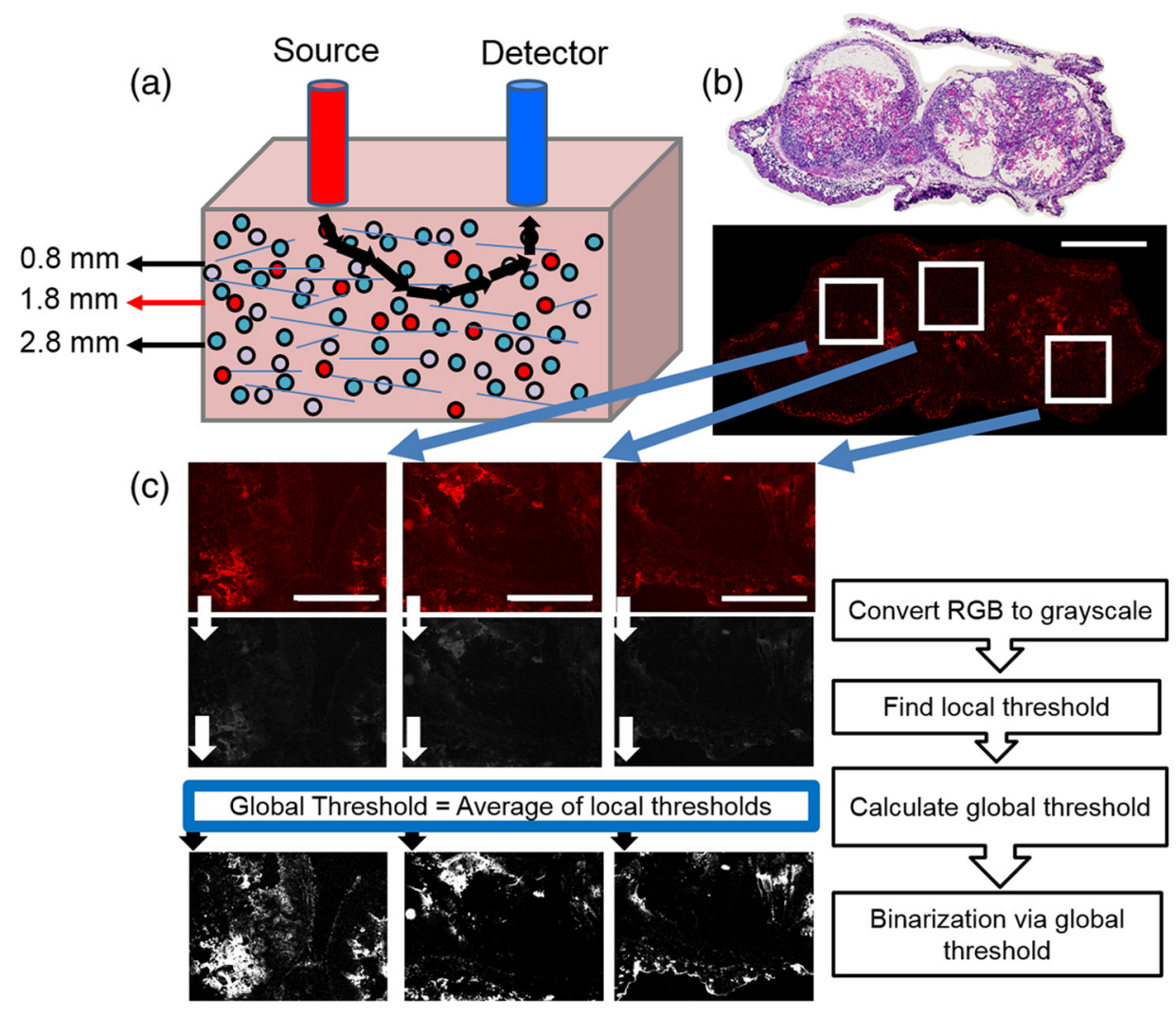

Fig. 2 (a) Schematic of optical probe placed on tissue indicating the three depths at which tumor slices were extracted for immunohistochemistry. (b) Stitched H\&E and pimonidazole immunofluorescence images of a tumor slice from a depth of $1.8 \mathrm{~mm}$. Scale bar corresponds to $1.8 \mathrm{~mm}$. (c) Flowchart describing the image analysis to calculate HF. Scale bar in these images corresponds to $600 \mu \mathrm{m}$. 

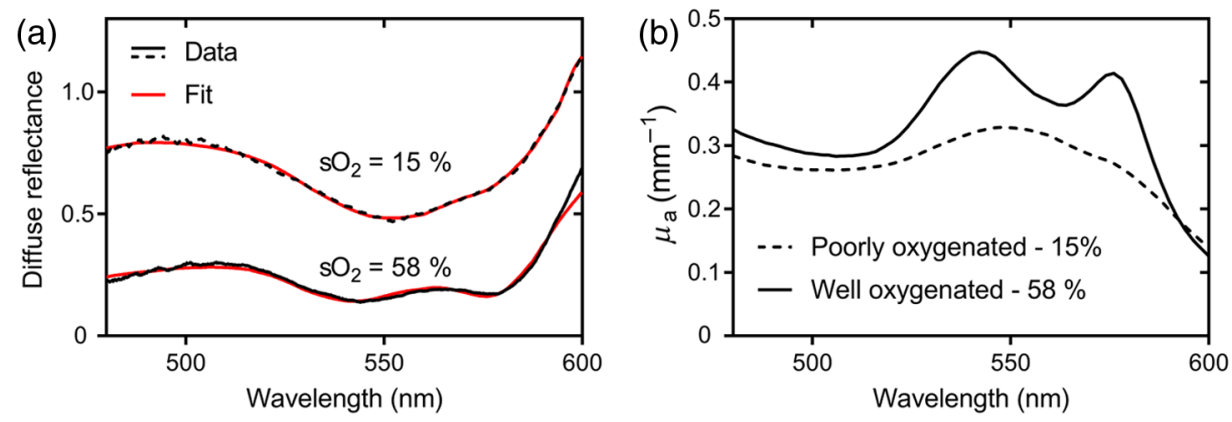

(c)

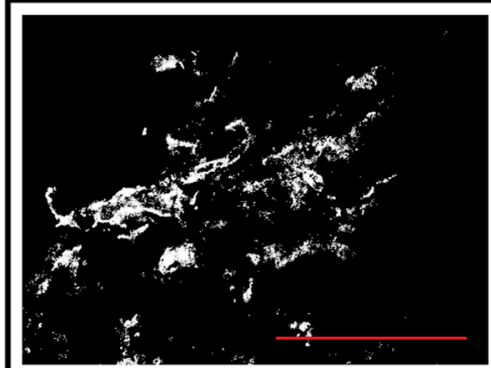

$\mathrm{sO}_{2}=58 \%$

$\mathrm{HF}=10.2 \%$

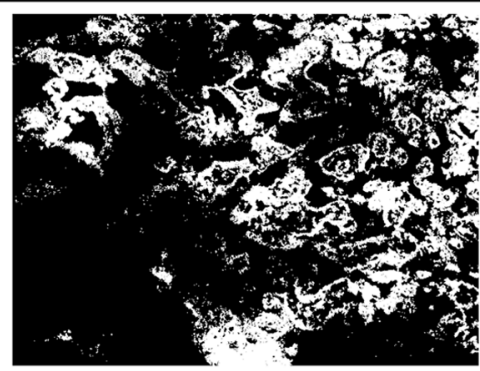

$\mathrm{SO}_{2}=15 \%$

$\mathrm{HF}=32.4 \%$

Fig. 3 (a) Representative in vivo DRS spectra and their LUT fits from UM-SCC-22B tumors with different levels of $\mathrm{sO}_{2}$. (b) Corresponding wavelength-dependent absorption coefficients determined from LUT fits to the spectra. (c) Processed pimonidazole immunofluorescence images from the same tumors. The calculated HF and vascular oxygenation are shown for each image. Scale bar corresponds to $600 \mu \mathrm{m}$.

We determined the association between the HF and the scattering and absorption properties of the UM-SCC-22B and UMSCC-47 tumors. We found a statistically significant negative correlation $(r=-0.50 ; p=0.02)$ between the $\mathrm{HF}$ and $\mathrm{sO}_{2}$
[Fig. 4(a)]. We also observed similar statistically significant correlations of the HF with THb [Fig. 4(b); $r=-0.45$; $p=0.04$ ] and $\mathrm{HbO}_{2}$ [Fig. 4(c); $r=-0.59 ; p=0.006$ ]. Taken together, these results indicate that measurements of
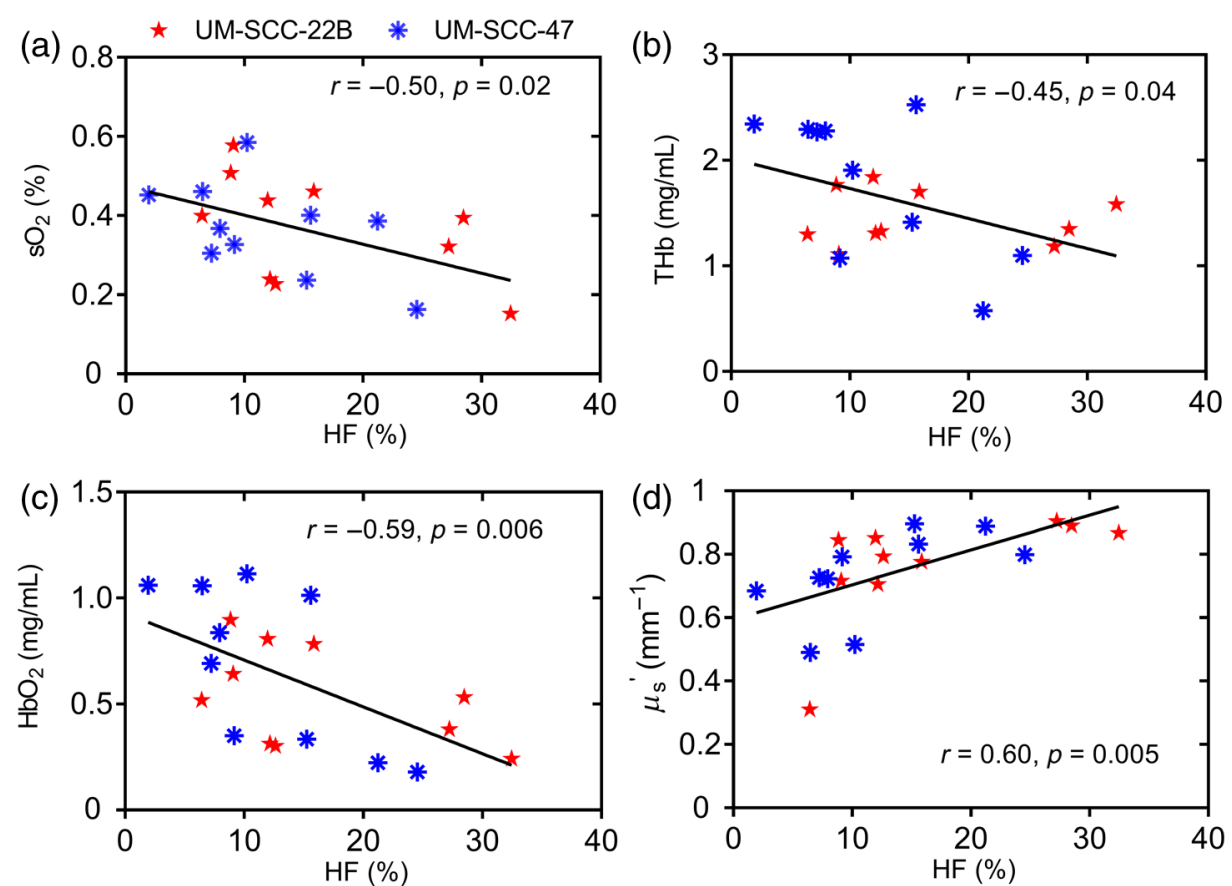

Fig. 4 Scatter plots representing the relationship between HF measured at a depth of $1.8 \mathrm{~mm}$ and DRSbased measures of (a) $\mathrm{sO}_{2}$, (b) $\mathrm{THb}$, (c) $\mathrm{HbO}_{2}$, and (d) mean $\mu_{\mathrm{s}}^{\prime}$ from UM-SCC-22B (red stars) and UM-SCC-47 (blue asterisks). Black solid line indicates the regression line. 


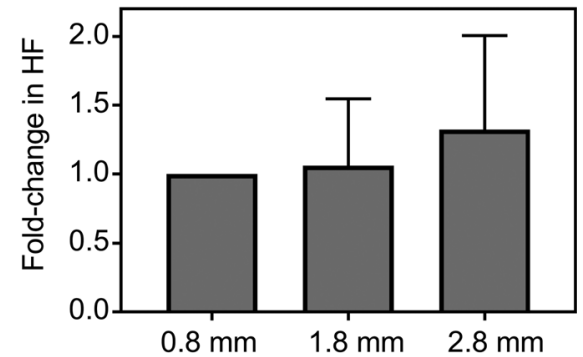

Fig. 5 Fold-change in tumor hypoxia levels with depth relative to the $\mathrm{HF}$ measured at $0.8 \mathrm{~mm}$. Error bars represent standard deviation of the mean.

vascular $\mathrm{sO}_{2}$ and $\mathrm{THb}$ can provide reliable estimates of the hypoxic state of tissue. Specifically, very low values of $\mathrm{sO}_{2}$ and $\mathrm{THb}$ can point to highly hypoxic and poorly perfused tumor.

In addition to evaluating hemoglobin-based parameters, we determined the relationship between $\mathrm{HF}$ and the mean reduced scattering coefficient [Fig. 4(d)]. We found a statistically significant positive correlation between the two parameters $(r=0.60$; $p=0.005)$. Since all of our tumors were excised at similar tumor volumes, this correlation is unlikely due to tumor volume. A previous study using optical spectroscopy by Vishwanath et al. found a highly significant positive correlation between mean reduced scattering coefficient and tumor necrotic fraction. ${ }^{20}$ Although we do not completely understand the relationship between HF and scattering in our study, it is possible that the highly hypoxic tumors also contained larger necrotic areas-the cellular and structural changes associated with tumor necrosis could potentially contribute to the increased scattering seen here. ${ }^{27,28}$ Our future studies include plans to transiently modify tumor hypoxia levels ${ }^{6}$ and measure the association between HF and tissue scattering and absorption. These studies could potentially shed further light on the basis for these interactions.

We also investigated the HF as a function of depth within each tumor. HF was calculated at additional depths of 0.8 and $2.8 \mathrm{~mm}$ from the surface of the tumor. For each tumor, the fold-change in $\mathrm{HF}$ at 1.8 and $2.8 \mathrm{~mm}$ was calculated with respect to the $\mathrm{HF}$ at $0.8 \mathrm{~mm}$. Although we observed a trend toward increasing HF with depth, this was not statistically significant (Fig. 5). These results are similar to a recent study using multispectral optoacoustic tomography on prostate cancer xenografts that found a well-oxygenated rim and a large decrease in oxygenation toward the tumor core. ${ }^{29}$ Furthermore, we did not find statistically significant correlations between optical properties and HFs at depths of 0.8 and $2.8 \mathrm{~mm}$ (data not shown). Although tumor xenografts are typically considered to be homogeneous, it is interesting to note a lack of correlation at depths that do not lie within the range of sampling depths of the probe.

In summary, we have demonstrated that the vascular oxygenation and total hemoglobin concentration measured using DRS are concordant with measurements of HF and can provide reliable indirect measures of tumor oxygenation. Monitoring changes in tumor oxygenation during therapy-radiation or chemo-can provide clinicians with a complementary aid to identify treatment response, and provide opportunities to investigate hitherto unknown reoxygenation kinetics in preclinical animal models in response to clinically relevant treatment regimens.

\section{Disclosures}

The authors have no financial interests or conflicts of interest in this manuscript.

\section{Acknowledgments}

This study was supported by the Arkansas Bioscience Institute and the University of Arkansas, College of Engineering.

\section{References}

1. M. Höckel et al., "Association between tumor hypoxia and malignant progression in advanced cancer of the uterine cervix," Cancer Res. 56, 4509-4515 (1996).

2. M. Nordsmark and J. Overgaard, "A confirmatory prognostic study on oxygenation status and loco-regional control in advanced head and neck squamous cell carcinoma treated by radiation therapy," Radiother. Oncol. 57, 39-43 (2000).

3. E. J. Hall and A. J. Giaccia, Radiobiology for the Radiologist, 7th ed., pp. 67-85, Lippincott, Williams, and Wilkins, Philadelphia, Pennsylvania (2012).

4. M. Höckel and P. Vaupel, "Tumor hypoxia: definitions and current clinical, biologic, and molecular aspects," J. Natl. Cancer Inst. 93, 266-276 (2001).

5. M. C. Kavanagh et al., "A comparison in individual murine tumors of techniques for measuring oxygen levels," Int. J. Radiat. Oncol. Biol. Phys. 44, 1137-1146 (1999).

6. J. A. Raleigh et al., "Comparisons among pimonidazole binding, oxygen electrode measurements, and radiation response in $\mathrm{C} 3 \mathrm{H}$ mouse tumors," Radiat. Res. 151, 580-589 (1999).

7. M. Nordsmark et al., "Measurements of hypoxia using pimonidazole and polarographic oxygen-sensitive electrodes in human cervix carcinomas," Radiother. Oncol. 67, 35-44 (2003).

8. P. Vaupel, D. K. Kelleher, and M. Höckel, "Oxygenation status of malignant tumors: Pathogenesis of hypoxia and significance for tumor therapy," Semin. Oncol. 28, 29-35 (2001).

9. L. H. Gray et al., "The concentration of oxygen dissolved in tissues at the time of irradiation as a factor in radiotherapy," Br. J. Radiol. 26, 638-648 (1953).

10. I. Tannock, "Response of aerobic and hypoxic cells in a solid tumor to adriamycin and cyclophosphamide and interaction of the drugs with radiation," Cancer Res. 42, 4921-4926 (1982).

11. J. M. Brown, "Tumor hypoxia in cancer therapy," Methods Enzymol. 435, 297-321 (2007).

12. D. M. Brizel et al., "Tumor oxygenation predicts for the likelihood of distant metastases in human soft tissue sarcoma," Cancer Res. 56, 941-943 (1996).

13. P. Vaupel et al., "Oxygenation of human tumors: evaluation of tissue oxygen distribution in breast cancers by computerized $\mathrm{O}_{2}$ tension measurements," Cancer Res. 51, 3316-3322 (1991).

14. K. Schlenger and P. Vaupel, "Oxygenation of carcinomas of the uterine cervix: evaluation by computerized $\mathrm{O} 2$ tension measurements," Cancer Res. 51, 6098-6102 (1991).

15. S. Matsumoto et al., "Low-field paramagnetic resonance imaging of tumor oxygenation and glycolytic activity in mice," J. Clin. Invest. 118, 1965-1973 (2008).

16. M. A. Varia et al., "Pimonidazole: a novel hypoxia marker for complementary study of tumor hypoxia and cell proliferation in cervical carcinoma," Gynecol. Oncol. 71, 270-277 (1998).

17. J. A. Raleigh et al., "Hypoxia and vascular endothelial growth factor expression in human squamous cell carcinomas using pimonidazole as a hypoxia marker," Cancer Res. 58, 3765-3768 (1998).

18. S. M. Evans et al., "Detection of hypoxia in human squamous cell carcinoma by EF5 binding," Cancer Res. 60, 2018-2024 (2000).

19. C. J. Koch, "Measurement of absolute oxygen levels in cells and tissues using oxygen sensors and 2-nitroimidazole EF5," Methods Enzymol. 352, 3-31 (2002).

20. K. Vishwanath et al., "Using optical spectroscopy to longitudinally monitor physiological changes within solid tumors," Neoplasia 11, 889-900 (2009). 
21. G. M. Palmer et al., "Quantitative diffuse reflectance and fluorescence spectroscopy: tool to monitor tumor physiology in vivo," J. Biomed. Opt. 14, 024010 (2012).

22. B. S. Nichols, N. Rajaram, and J. W. Tunnell, "Performance of a lookup table-based approach for measuring tissue optical properties with diffuse optical spectroscopy," J. Biomed. Opt. 17, 057001 (2012).

23. N. Rajaram, T. H. Nguyen, and J. W. Tunnell, "Lookup table-based inverse model for determining optical properties of turbid media," J. Biomed. Opt. 13, 050501 (2008).

24. N. Rajaram et al., "Experimental validation of the effects of microvasculature pigment packaging on in vivo diffuse reflectance spectroscopy," Lasers Surg. Med. 42, 680-688 (2010).

25. G. E. Arteel et al., "Evidence that hypoxia markers detect oxygen gradients in liver: pimonidazole and retrograde perfusion of rat liver," Br. J. Cancer 72, 889-895 (1995).

26. N. A. Otsu, "Threshold selection method from gray-level histograms," IEEE Trans. Syst. Man. Cybern. 9, 62-66 (1979).

27. R. H. Thomlinson and L. H. Gray, "The histological structure of some human lung cancers and the possible implications for radiotherapy," Br. J. Cancer 9, 539-549 (1955).

28. R. Drezek et al., "Light scattering from cervical cells throughout neoplastic progression: influence of nuclear morphology, DNA content, and chromatin texture," J. Biomed. Opt. 8, 7 (2003).

29. M. R. Tomaszewski et al., "Oxygen enhanced optoacoustic tomography (OE-OT) reveals vascular dynamics in murine models of prostate cancer," Theranostics 7, 2900-2913 (2017).
Sina Dadgar received his BS and MS degrees in electrical engineering from Azad University, Iran, 2012, and Bahçeşehir University, Turkey, 2015, respectively. He is currently a second year PhD student in biomedical engineering at the University of Arkansas. His research focus is on utilizing optical spectroscopy to differentiate therapy responders versus nonresponders in human head and neck cancer.

Joel Rodríguez Troncoso is currently a biomedical engineering senior at the University of Arkansas, Fayetteville. His research interests include diffuse reflectance spectroscopy, tumor response to radiation, and finding functional markers in breast tumors to differentiate between indolent and aggressive disease.

Narasimhan Rajaram is an assistant professor of biomedical engineering at the University of Arkansas, Fayetteville. He received his $\mathrm{PhD}$ in biomedical engineering from the University of Texas at Austin and completed postdoctoral training at Duke University. His research is focused on the development of functional and molecular optical imaging strategies that can survey the tumor microenvironment and determine early response to therapy as well as long-term clinical outcome in terms of tumor recurrence and metastasis. 